\title{
Erratum: All orders results for self-crossing Wilson loops mimicking double parton scattering
}

\section{Lance J. Dixon ${ }^{a, b}$ and Ilya Esterlis ${ }^{a}$}

${ }^{a} S L A C$ National Accelerator Laboratory, Stanford University,

Stanford, CA 94309, U.S.A.

${ }^{b}$ Walter Burke Institute for Theoretical Physics, California Institute of Technology, 1200 E. California Blvd, Pasadena, CA 91125, U.S.A.

E-mail: lance@slac.stanford.edu, ilyae@stanford.edu

ERRATUM TO: JHEP07(2016)116

ARXIV EPRINT: 1602.02107

1. Equations (D.14)-(D.17) were computed from an incorrect eq. (D.13), the correct equation is

$$
\begin{aligned}
\rho \equiv \frac{\mathcal{A}_{6}^{\mathrm{NMHV}}}{\mathcal{A}_{6}^{\mathrm{BDS}-\text { like }}}= & \frac{1}{2\left(1+w^{*}\right)}[E(u, v, w)+\tilde{E}(u, v, w)+E(w, u, v)-\tilde{E}(w, u, v)] \\
& +\frac{w^{*}}{2\left(1+w^{*}\right)}[E(v, w, u)+\tilde{E}(v, w, u)+E(w, u, v)-\tilde{E}(w, u, v)] .
\end{aligned}
$$

In the original version a complex conjugation and factor of $1 / 2$ were missing. Equations (D.14)-(D.17) below are the corrected versions, based on the corrected (D.13) above. Also, in the original text, equations (D.14)-(D.17) were intended to be for $2 \rightarrow 4$ kinematics with $v \rightarrow 0^{+}$. However, the $v \rightarrow 0^{-}$limit of $3 \rightarrow 3$ kinematics is simpler, having no double discontinuity. The corrected equations (D.14)-(D.17) below are analytically continued to $3 \rightarrow 3$ kinematics by the method discussed in section 3.1 of the main text, and then the limit $v \rightarrow 0^{-}$is taken:

$$
\begin{aligned}
\rho^{(1)}= & -\frac{1}{2} \ln ^{2}|v|+2 \zeta_{2}+\pi i\left[\frac{1+w}{1+w^{*}}+1\right], \\
\rho^{(2)}= & \frac{1}{8} \ln ^{4}|v|-\frac{1}{2} \zeta_{2} \ln ^{2}|v| \\
& +\pi i\left[\frac{1+w}{1+w^{*}}\left(-\frac{1}{2} \ln ^{2}|\delta|+\ln |\delta|+\zeta_{2}-1\right)-\frac{1}{2} \ln ^{2}|v|-\ln |v|-\zeta_{3}+\zeta_{2}-1\right],
\end{aligned}
$$




$$
\begin{aligned}
& \rho^{(3)}=-\frac{1}{48} \ln ^{6}|v|-\frac{1}{4} \zeta_{4} \ln ^{2}|v|+\frac{91}{12} \zeta_{6} \\
&+\pi i[ \frac{1+w}{1+w^{*}}\left(\frac{1}{8} \ln ^{4}|\delta|-\frac{1}{2} \ln ^{3}|\delta|+\frac{3}{2} \ln ^{2}|\delta|-\left(\zeta_{3}+3\right) \ln |\delta|+\frac{1}{2} \zeta_{4}+\zeta_{3}+3\right) \\
&+\frac{1}{8} \ln ^{4}|v|+\frac{1}{2} \ln ^{3}|v|-\frac{1}{2}\left(\zeta_{3}-3\right) \ln ^{2}|v|+\left(\zeta_{3}+3\right) \ln |v| \\
&\left.+7 \zeta_{5}-3 \zeta_{2} \zeta_{3}+\frac{1}{2} \zeta_{4}+\zeta_{3}+3\right], \\
& \rho^{(4)}=\frac{1}{384} l^{8}|v|+\frac{1}{48} \zeta_{2} \ln ^{6}|v|+\frac{7}{16} \zeta_{4} \ln ^{4}|v|+\left(\frac{13}{48} \zeta_{6}+\frac{1}{2}\left(\zeta_{3}\right)^{2}\right) \ln ^{2}|v|-\frac{1325}{36} \zeta_{8}-2 \zeta_{2}\left(\zeta_{3}\right)^{2} \\
&+\pi i\left[\frac { 1 } { 1 + w } \left(-\frac{1}{48} \ln ^{6}|\delta|+\frac{1}{8} \ln ^{5}|\delta|-\frac{1}{8}\left(\zeta_{2}+5\right) \ln ^{4}|\delta|+\left(-\frac{1}{6} \zeta_{3}+\frac{1}{2} \zeta_{2}+\frac{5}{2}\right) \ln ^{3}|\delta|\right.\right. \\
&-\left(\frac{7}{4} \zeta_{4}-\frac{1}{2} \zeta_{3}+\frac{3}{2} \zeta_{2}+\frac{15}{2}\right) \ln ^{2}|\delta|+\left(4 \zeta_{5}-3 \zeta_{2} \zeta_{3}+\frac{7}{2} \zeta_{4}-\zeta_{3}+3 \zeta_{2}+15\right) \ln |\delta| \\
&\left.-\frac{13}{24} \zeta_{6}-2\left(\zeta_{3}\right)^{2}-4 \zeta_{5}+3 \zeta_{2} \zeta_{3}-\frac{7}{2} \zeta_{4}+\zeta_{3}-3 \zeta_{2}-15\right) \\
&-\frac{1}{48} \ln ^{6}|v|-\frac{1}{8} \ln ^{5}|v|+\left(\frac{3}{8} \zeta_{3}-\frac{1}{8} \zeta_{2}-\frac{5}{8}\right) \ln ^{4}|v|+\left(\frac{1}{12} \zeta_{3}-\frac{1}{2} \zeta_{2}-\frac{5}{2}\right) \ln ^{3}|v| \\
&+\left(\frac{25}{8} \zeta_{5}+2 \zeta_{2} \zeta_{3}-\frac{7}{4} \zeta_{4}+\frac{1}{4} \zeta_{3}-\frac{3}{2} \zeta_{2}-\frac{15}{2}\right) \ln ^{2}|v| \\
&+\left(-\frac{3}{2}\left(\zeta_{3}\right)^{2}-5 \zeta_{5}+\zeta_{2} \zeta_{3}-\frac{7}{2} \zeta_{4}+\frac{1}{2} \zeta_{3}-3 \zeta_{2}-15\right) \ln |v| \\
&-\frac{1381}{32} \zeta_{7}+\frac{43}{2} \zeta_{2} \zeta_{5}+4 \zeta_{3} \zeta_{4}-\frac{13}{24} \zeta_{6}-\frac{5}{2}\left(\zeta_{3}\right)^{2}-5 \zeta_{5}+\zeta_{2} \zeta_{3} \\
&\left.-\frac{7}{2} \zeta_{4}+\frac{1}{2} \zeta_{3}-3 \zeta_{2}-15\right] \cdot \\
&
\end{aligned}
$$

2. The discussion immediately following equations (D.14)-(D.17) was based on the original, incorrect version of these equations. The main point originally read:

"We see that there are indeed logarithmically singular $\ln \delta$ terms in the imaginary part and in the double discontinuity $(2 \pi i)^{2}$ term, beginning at two loops. However, there are no $\ln \delta$ terms in the part with the $\left(1+w^{*}\right) /(1+w)$ prefactor; that is, the terms depending on the azimuthal component of the vector $\vec{z}$ in eq. (2.22) are finite."

The correct version is:

"We see that there are indeed logarithmically singular $\ln |\delta|$ terms in the imaginary part beginning at two loops. However, the $\ln |\delta|$ terms appear only in the part with the $(1+w) /\left(1+w^{*}\right)$ prefactor; that is, the terms that are independent of the azimuthal component of the vector $\vec{z}$ in eq. (2.22) are finite."

Open Access. This article is distributed under the terms of the Creative Commons Attribution License (CC-BY 4.0), which permits any use, distribution and reproduction in any medium, provided the original author(s) and source are credited. 